\title{
Diplomacia da saúde global: proposta de modelo conceitual'
}

\section{Global health diplomacy: conceptual framework proposal}

\section{Pollyanna Martins}

Secretaria de Saúde do Estado do Ceará. Fortaleza, CE, Brasil.

E-mail: pollysobralळyahoo.com.br

\section{Andréa Silvia Walter de Aguiar}

Universidade Federal do Ceará. Fortaleza, CE, Brasil.

E-mail: aswaguiarðpq.cnpq.br

\section{Caroline Antero Machado Mesquita}

Faculdade Maurício de Nassau. Fortaleza, CE, Brasil.

E-mail: carolantero4l@gmail.com

\section{Francisca Jamila Ricarte Alexandrino}

Universidade Federal do Ceará. Fortaleza, CE, Brasil.

E-mail: jamilaricartealexandrinoळgmail.com

\section{Nayane Cavalcante Ferreira da Silva}

Faculdade Maurício de Nassau. Fortaleza, CE, Brasil.

E-mail: nayanecferreiraœgmail.com

\section{Melinna dos Santos Moreno}

Universidade Federal do Ceará. Programa de Pós-Graduação em Odontologia. Fortaleza, CE, Brasil.

E-mail: melinnamoreno®hotmail.com

\section{Correspondência}

Andréa Sílvia Walter de Aguiar

Rua Monsenhor Furtado, s/n, Rodolfo Teófilo.

Fortaleza, CE, Brasil. CEP 60430-355.

\section{Resumo}

Este estudo busca construir, a partir das publicações sobre diplomacia da saúde global (DSG), representação deste campo de conhecimento, seus principais conceitos e características. A partir de uma pesquisa bibliográfica na base de dados PubMed, fez-se uma busca integrada das produções científicas publicadas até agosto de 2016. Os descritores identificados e critérios de inclusão adotados selecionaram 54 publicações que compuseram a amostra final. Os dados quantitativos foram analisados por estatística descritiva, e as informações qualitativas, pela análise de conteúdo temática. As publicações sobre o tema tiveram início em 1970, mas 85,2\% abrangem o período de 2007 a 2014. A partir da análise de conteúdo dos resultados das produções científicas, foi elaborado um modelo teórico conceitual que abrange os seguintes macrotemas da DSG: conceito de diplomacia da saúde; uso da diplomacia na cooperação internacional em pesquisa; diplomacia da saúde como ferramenta para fortalecer a política externa; estratégias dos países para fortalecer a DSG e cooperação internacional entre os países; diplomacia da saúde fortalecendo a saúde global; saúde como um bem comum entre as nações em um período de globalização e desafios da diplomacia da saúde para fortalecer a saúde global. A DSG compreende estratégias dos países e cooperação internacional para fortalecer a saúde global e é uma ferramenta para fortalecer a política externa das nações. Promover a cooperação internacional em pesquisa, capacitar recursos humanos nessa área e emancipar países emergentes com base nas intervenções realizadas constituem grandes desafios da DSG. Palavras-chave: Saúde Mundial; Cooperação Internacional; Relações Internacionais.

1 Este artigo é resultado de pesquisa denominada "Diplomacia da saúde nas ações e serviços de saúde bucal na faixa de fronteira brasileira", financiada pelo CNPq, por meio do Edital Universal (14/2014). 


\section{Abstract}

The aim of this article is to build, from the publications on global health diplomacy (GHD), a representation of this field of knowledge, its main concepts and features. We searched the literature in PubMed database for publications on the subject until August 2016. The keywords and inclusion criteria selected 54 publications that composed the final sample. Quantitative data were analyzed using descriptive statistics, and qualitative information, by the thematic content analysis. Publications on the subject began in 1970, but $85.2 \%$ of the studies cover the period from 2007 to 2014. With the content analysis of the results of this scientific production, we produced a conceptual theoretical model covering the following subjects of GHD: health diplomacy concept; use of diplomacy in international cooperation with research; health diplomacy as a tool for strengthening foreign policy; strategies for strengthening the diplomacy of global health and international cooperation among countries; health diplomacy strengthening global health; health as a common good among the nations in globalization; health diplomacy challenges to strengthen global health. GHD comprises country strategies and international cooperation to strengthen global health and is a tool for strengthening the foreign policy of nations. The major challenges of GHD are to promote international cooperation in research, train human resources from this area and emancipate emerging countries based on ongoing interventions.

Keywords: World Health; International Cooperation; International Relations.

\section{Introdução}

Ministros e autoridades sanitárias de 194 países participaram da $68^{\mathrm{a}}$ Assembleia Mundial da Saúde, da Organização Mundial da Saúde $(\mathrm{OMS})^{2}$, realizada de 18 a 26 de maio de 2015, em Genebra, Suíça. A incorporação da saúde na agenda para o desenvolvimento pós-2015 e o impacto da contaminação ambiental na saúde estavam entre os temas debatidos.

Esse conjunto de negociações desenvolvidas em diversos níveis, que delineia e gerencia o ambiente das políticas globais em saúde e que, idealmente, produz melhores resultados para a saúde da população de cada país envolvido e reforça o compromisso de um amplo arco de atores em prol do empreendimento comum de assegurar a saúde como direito humano e bem público, tem sido denominado "diplomacia da saúde global” (DSG) (Kicksbusch et al., 2007). Trata-se de um campo novo de conhecimento e prática, cujo objeto é a saúde e as negociações internacionais em torno desta, envolvendo diferentes disciplinas e profissionais de diversos perfis, como diplomatas e profissionais de saúde (Buss, 2013).

A DSG é uma disciplina emergente em que a saúde é incorporada à diplomacia tradicional como uma ferramenta de política externa (Kicksbusch et al., 2007). A DSG é usada por muitos países para melhorar as relações de confiança entre os estados, a saúde nacional e mundial. Nessa perspectiva, os ministérios da saúde passaram a desempenhar um papel duplo: promover a saúde do país e a saúde global (Fourie, 2013).

Objetiva-se propor, a partir das publicações sobre DSG, representação deste campo de conhecimento, seus principais conceitos e características, assim como apontar novas questões que carecem de pesquisas.

\section{Metodologia}

Para construir o modelo conceitual, optou-se por uma pesquisa bibliométrica embasada em dados publicados e usada para mensurar as características de determinados temas, o que de certa forma indica sua produção científica (Vanti, 2002).

2 WHO - WORLD HEALTH ORGANIZATION. 68th World Health Assembly. Disponível em: <https://goo.gl/Lbuk71>. Acesso em: 2 maio 2015. 
A base de dados consultada foi a U.S. National Library of Medicine (PubMed), em que se fez uma busca integrada das produções científicas publicadas até agosto de 2016. Os descritores adotados foram identificados pelo Medical Subject Headings (MeSH) dessa base de dados. Na busca, foram encontrados descritores que se apresentaram como sinônimos do tema pesquisado, visto que o termo "diplomacia da saúde" não foi encontrado no MeSH. Os descritores identificados e seus respectivos sinônimos foram:

1. Saúde Mundial/World Health/Salud Mundial. Sinônimos: Problemas Internacionais de Saúde, Saúde Global;

2. Cooperação Internacional/International Cooperation/Cooperación Internacional. Sinônimos: Auxílio Internacional, Ajuda Externa;

3. Internacionalidade/Internationality/Internacionalidad. Sinônimos: Relações Internacionais.

A estratégia de busca adotou os seguintes descritores no campo de busca avançada da PubMed: "world health" [MeSH Terms] or "world" [All Fields] and "health" [All Fields] or "world health" [All Fields] and "international cooperation" [MeSH Terms] or "international" [All Fields] and "cooperation" [All Fields] or "international cooperation" [All Fields] and "internationality" [MeSH Terms] or "internationality" [All Fields] and "health" [MeSH Terms] or "health" [All Fields] and "diplomacy" [All Fields]. Os critérios de inclusão para a seleção da amostra em relação à disponibilidade de texto foram: "Free full text", "Full text".

Foram encontradas 54 publicações que compuseram a amostra final. 0 material foi submetido à pré-análise, e, pela leitura integral do texto, foram categorizados segundo as seguintes variáveis: $1^{0}$ autor, ano de publicação, periódico, título, objetivo, resultado principal relativo ao tema, tipo de publicação, universidade/instituição do $1^{0}$ autor e país de publicação do periódico. Na variável "tipo de publicação" foram considerados como editoriais os comentários, editoriais, debates e artigos de opinião publicados no período. Os resultados foram transcritos em uma planilha eletrônica no Microsoft Excel, os dados quantitativos foram analisados por estatística descritiva simples, e os dados qualitativos, pela análise temática de conteúdo.
A análise de conteúdo temática foi feita para descobrir os "núcleos de sentido" que compõem a comunicação e cuja presença ou frequência pode significar alguma coisa para o tema em estudo (Bardin, 2011). Na coleta dos dados qualitativos (título, objetivos, resultados principais) extraídos da leitura dos textos, foram usadas frases e parágrafos como unidades de registro, codificadas em temas reagrupadas em categorias ou subcategorias.

\section{Resultados}

As publicações sobre o tema iniciaram em 1970. Todavia, a partir de 2007 observou-se maior frequência ( $85,2 \%$ abrangem o período de 2007 a 2014). A maioria das instituições ou universidades às quais estão vinculados os primeiros autores dos artigos localiza-se nos Estados Unidos da América (61\%), na Suíça $(14,8 \%)$ e no Brasil (5,6\%). Na Suíça destacaram-se a World Health Organization, de Genebra, e o Graduate Institute for International Development Studies, da mesma cidade; nos EUA, organizações não governamentais, departamentos estatais, Columbia University, Indiana University e University of California; no Brasil, a Fundação Oswaldo Cruz. Entretanto, não se observou semelhante tendência quando se considerou o país de publicação do periódico; neste caso, os EUA (53,7\%) e o Reino Unido $(25,9 \%)$ lideraram o total de publicações.

Quanto ao tipo de publicação, destacaram-se os editoriais (38,9\%), seguidos de artigos de revisão/ reflexão (29,6\%) e artigos originais/estudo de caso (22,2\%). Artigos teóricos conceituais totalizaram 9,3\% das publicações. Os resultados principais das pesquisas sobre o tema foram submetidos à análise de conteúdo temática e, depois do processo de codificação e categorização, emergiram sete macrotemas: conceito de diplomacia da saúde; uso da diplomacia na cooperação internacional em pesquisa; diplomacia da saúde como ferramenta para fortalecer a política externa; estratégias dos países para fortalecer a DSG e cooperação internacional entre os países; diplomacia da saúde fortalece a saúde global; a saúde como um bem comum entre as nações em um período de globalização e desafios da diplomacia da saúde para fortalecer a saúde global. 


\section{Discussão}

A produção científica sobre DSG é recente, e o tema ainda é pouco conhecido nas comunidades acadêmicas, o que foi confirmado pela predominância de editoriais entre as produções científicas selecionadas. A função de um editorial em um periódico é discutir temas atuais e controversos e publicar análises ou opiniões sobre um tema importante. A abordagem qualitativa com base em estudos de caso e os artigos de revisão que refletem sobre o tema ou têm a função de introduzir conceitos sobre determinado campo de conhecimento endossam essa premissa.

A inserção dos autores que pesquisam e escrevem sobre o tema está concentrada nos EUA e na
Suíça, o que pode ser explicado pelo fato de esses dois países serem os líderes nas questões diplomáticas na área da saúde, pois sediam a Organização das Nações Unidas (ONU) e OMS, respectivamente.

Com base na análise dos resultados das pesquisas sobre o tema, elaborou-se uma árvore da DSG, e suas raízes representam os conceitos e as teorias sobre o tema; os galhos, as consequências da diplomacia; e os frutos, os produtos gerados (Figura 1).

A respeito dos principais resultados das pesquisas sobre o tema, discutem-se a seguir os macrotemas que emergiram do processo de codificação e categorização dos dados qualitativos e que compõem a árvore da DSG.

Figura I - Modelo conceitual: árvore da diplomacia da saúde global

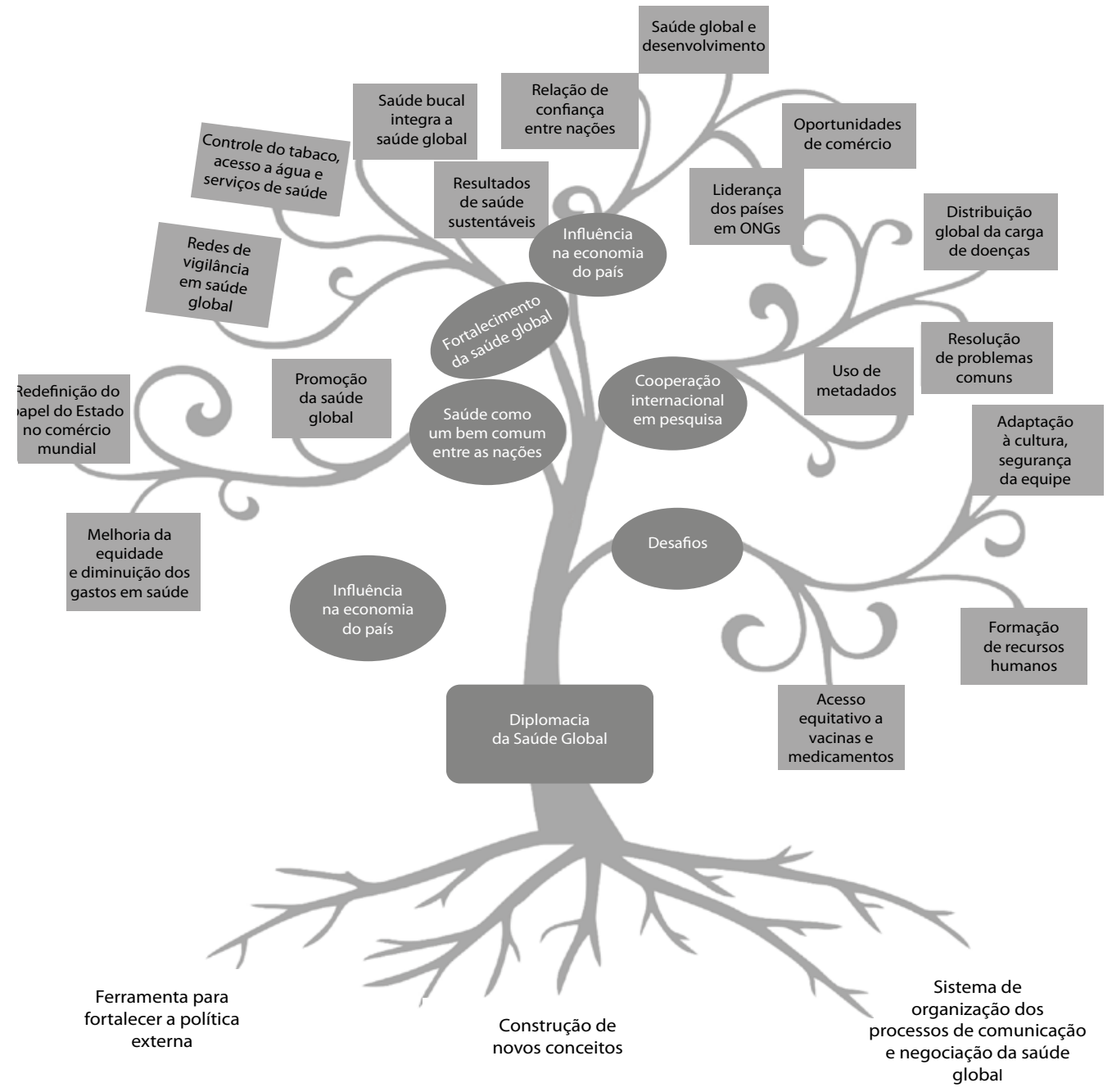




\section{Evolução conceitual da Diplomacia da Saúde Global}

Dentro da tipologia da diplomacia pública, a DSG é um conceito autônomo, um instrumento de política externa que não recorre aos tradicionais meios e canais diplomáticos para atingir seus objetivos definidos. Ela assume características próprias que a distingue de outros conceitos que lhe são próximos, como a diplomacia tradicional, e seu conceito evoluiu ao longo do tempo, conforme o quadro a seguir.

Embora a emergência do termo "DSG” seja recente, a interação entre diplomacia e saúde não é uma prática contemporânea entre as nações. "Os registros diplomáticos do Egito antigo que datam de 29 séculos atrás identificam os médico-sacerdotes do Nilo que acompanhavam as missões egípcias para países vizinhos" (Badeau, 1970, p. 303).

Nos períodos pós-guerra, a saúde constituiu-se em um objetivo comum entre as nações, e isso passou a se refletir nas ações diplomáticas. A expansão da comunicação pública (rádio, televisão, imprensa etc.) favoreceu a difusão de assuntos nacionais e internacionais. A libertação de colônias, a educação popular e a conscientização política aumentaram a pressão por acesso à educação e à saúde nos países emergentes e gerou novas demandas para as ações diplomáticas (Badeau, 1970). Intensificaram-se as ações humanitárias diplomáticas na área da saúde, o que contribuiu para a inclusão como uma ferramenta de política externa dos países.

French (1992) introduziu o termo "diplomacia internacional" no que diz respeito aos esforços e à preocupação das nações com as questões ambientais. Sharpless (1995) considerou que as relações diplomáticas funcionam como agentes de modificação comportamental nos países em desenvolvimento. Ao analisar os primeiros cem anos de diplomacia da saúde internacional, mostrou-se a vulnerabilidade da governança global e os riscos que a globalização causou à saúde pública, refletindo sobre a necessidade da cooperação internacional para controlá-los.

A partir das premissas anteriores e considerando que a saúde internacional depende do melhor controle de doenças em nível nacional ou local (Hardiman, 2003), nasce o termo "DSG", definido como um conjunto de negociações desenvolvidas em diversos níveis que delineia e gerencia o ambiente das políticas globais em saúde e que, idealmente, produz melhores resultados para a saúde da população de cada país envolvido, bem como reforça o compromisso de um amplo arco de atores em prol da garantia da saúde como direito humano e bem público (Kickbusch et al., 2007). Entretanto, Drager e Fidler (2007) assinalaram o desafio de alinhar os interesses nacionais, a política externa e a DSG em um mundo globalizado, com distintas realidades epidemiológicas e éticas.

\section{Quadro I - Evolução conceitual de Diplomacia da Saúde Global ao longo do tempo}

\begin{tabular}{|l|l|}
\hline Ano & Macrotema \\
\hline 1970 & Diplomacia médica - ações humanitárias (Badeau, 1970) \\
\hline 1992 & Diplomacia internacional - questões ambientais (French, 1992) \\
\hline \multirow{2}{*}{2001} & Efeitos da globalização na saúde (Fidler, 200I) \\
\hline \multirow{2}{*}{2003} & Saúde global dependente da saúde de cada nação (Hardiman, 2003) \\
\hline \multirow{2}{*}{2007} & DSG - negociações entre nações para promover a saúde global (Kickbusch et al., 2007) \\
\cline { 2 - 3 } & DSG - ferramenta para fortalecer a política externa (Drager; Fidler, 2007) \\
\hline \multirow{2}{*}{2008} & DSG - participação das ONGs em negociações na promoção da saúde global (Adams; Novotny; Leslie, 2008) \\
\hline \multirow{2}{*}{2010} & DSG - parcerias entre ONGs e governos (Kosaraju et al., 20ıo) \\
\hline \multirow{2}{*}{2013} & DSG - melhora da saúde global, equidade, relações de confiança entre estados (Kickbusch; Kökény, 2013) \\
\cline { 2 - 3 } & DSG - políticas e programas que geram resultados de saúde sustentáveis (Ratzan, 2013) \\
\hline
\end{tabular}


Autores acrescentaram que a DSG consiste na participação de organizações não governamentais (ONGs) em programas de saúde internacionais que abrangem a globalização da ciência e da pesquisa farmacêutica, bem como o uso de línguas militarizadas de biossegurança para reformular programas de saúde pública (Adams; Novotny; Leslie, 2008). Kosaraju et al. (2010) complementaram esse conceito ao afirmarem que a parceria entre governos e ONG cria infraestrutura sustentável para ações de saúde global. Assim, a saúde pode interagir com a política externa como instrumento para atingir outras metas, podendo ser empregada para atingi-las na diplomacia da saúde e influenciando a arena multilateral da saúde (Kickbusch, 2011).

Complementou-se esse conceito ao apontar a influência da saúde na economia dos países. As inter-relações na saúde global podem se tornar um conflito para as relações externas dos países-membros de uma região ou bloco econômico, comercial ou político. No contexto da globalização, a saúde está intimamente ligada ao meio ambiente, ao comércio, ao crescimento econômico, ao desenvolvimento social, à segurança nacional e aos direitos humanos (Bernardini-Zambrini; Pedrosa; Sotelo, 2011).

A DSG resulta em melhoria da saúde global, equidade e relações de confiança entre estados. Os elementos que contribuíram para a ascensão da diplomacia foram os acordos comerciais, pandemias, maior coerência entre a política externa e interna de saúde (Kickbusch; Kökény, 2013). Esses autores postularam que a DSG refere-se a um sistema de organização dos processos de comunicação e negociação que moldam o ambiente político global em matéria de saúde e seus determinantes.Nessa perspectiva, a saúde faz parte das ações diplomáticas da ONU, do G8, do G20, do BRICS, da União Europeia e dos ministérios da saúde que passaram a desempenhar um papel duplo: promover a saúde do país e a saúde global.

A DSG é o processo de se engajar, motivar e comunicar múltiplas partes para desenvolver políticas e programas que promovam resultados de saúde sustentáveis (Ratzan, 2013). O principal objetivo da DSG é promover a saúde global, entretanto, algumas questões que são comuns às nações, como os acordos internacionais e metas para atenção às doenças crônicas, os acordos entre indústrias para diminuírem teores de açúcar e sal nos alimentos, a inserção e contribuição de subáreas da saúde na saúde global, como a saúde bucal, e o papel desses atores na DSG ainda são pouco discutidos na bibliografia acadêmica.

No caso da saúde bucal, Garcia e Tabak (2011) consideraram que a saúde oral integra a saúde global em macrotemas, como o controle do tabaco, a água potável, a saúde materna e infantil e a pesquisa sobre os sistemas de saúde. Entretanto, a efetividade dessas intervenções e acordos bem como as responsabilidades dos países e governos com suas implementações permanecem questionáveis. Para enfrentar essa complexidade de inter-relações, que afeta globalmente a saúde das populações em diversas partes do mundo, são necessárias intervenções, políticas específicas e suficiente produção de conhecimentos e capacitação de recursos humanos nessa área (Almeida, 2010).

\section{Diplomacia da saúde na cooperação internacional em pesquisa}

Fortaleceu-se o uso de metadados na análise de distribuição global da doença, que podem ser basicamente definidos como "dados que descrevem dados”, ou seja, informações úteis para identificar, localizar, compreender e gerenciá-los. Eles podem ajudar na agenda pós-2015 a partir de um espaço de intervenção para integrar a ciência com seu contexto sociotécnico (Özdemir et al., 2014).

Em consonância, Fedoroff (2009) destacou que colaborações científicas entre as nações para enfrentar problemas comuns e as interações científicas internacionais podem contribuir para diminuir o fosso digital (acesso desigual à ciência e à tecnologia) e o acesso desigual a comida, água, energia, saúde e desenvolvimento econômico.

A cooperação científica internacional é um fator determinante para superar as desigualdades entre os países, geradas pela transição epidemiológica, pela carga de doença desigual entre os países e pela pobreza, podendo colaborar para a governança da saúde global colaborativa e resolver problemas de saúde comuns das nações, por meio de metadados para priorizar intervenções com base no conheci- 
mento da distribuição global da carga de doenças (Novotny, 2006).

Um dos desafios para fortalecer a cooperação internacional no desenvolvimento de pesquisas em saúde global é melhorar o alinhamento e a harmonização do financiamento das investigações de acordo com as necessidades dos países beneficiários (Røttingen et al., 2009).

\section{Diplomacia da saúde como ferramenta para for- talecer a política externa}

Barraclough e Phua (2007) apontaram que as ameaças das doenças infecciosas à saúde humana e à atividade econômica causaram intensificação e formalização organizacional da DSG tanto em nível nacional quanto internacional.

A DSG tem sido uma pedra angular da política externa cubana desde o início da revolução, há cinquenta anos. Cuba começou a conduzir a diplomacia da saúde em 1960, mediante o envio de uma equipe médica para o Chile, a fim de fornecer socorro depois de um grande terremoto. Doravante, Cuba prestou assistência médica a mais de cem países em todo o mundo, tanto em situações de emergência a curto prazo quanto a longo prazo (Feinsilver, 2010). Exemplos recentes dessas iniciativas são a Missión Barrio Adentro, na Venezuela, e o Programa Mais Médicos, no Brasil.

Além de ser utilizada para promover a saúde global, os países menos desenvolvidos utilizam a DSG para fortalecer sua liderança em organismos internacionais, como a ONU, gerar oportunidades comerciais e melhorar a relação de confiança com outras nações (Kickbusch; Kökény, 2013). Em concordância, Seiff (2013) reforçou essa afirmação ao constatar que a liderança da Indonésia no campo da DSG contribuiu para que conseguisse diplomaticamente a manutenção da Association of Southeast Asian Nations (Asean), além de ocupar cargos na ONU e no Fundo Global contra aids, malária e tuberculose.

A divulgação do National Health System, pela Inglaterra, contribuiu para o aprendizado da implantação de sistemas de saúde com cobertura universal em países emergentes, além de gerar oportunidades comerciais (Chalkidou; Vega, 2013).
ADSG, adotada como ferramenta para fortalecer a política externa das nações, parte da premissa de que a saúde influencia a economia dos países e que a saúde global é parte do bem-estar e do desenvolvimento, em particular dos países menos desenvolvidos, além de colaborar para a liderança de países em organismos internacionais, gerando oportunidades comerciais e melhorando a relação de confiança entre as nações.

\section{Estratégias dos países para fortalecer a DSC e a cooperação internacional}

Cahill (1997) postulou que a saúde e as situações de conflito e desastre são as bases para a intervenção internacional, devendo-se adotar medidas diplomáticas, sobretudo nos países menos desenvolvidos, à época denominados "de terceiro mundo". Nessa perspectiva, o Brasil tem um papel ativo na prestação de ajuda para o desenvolvimento de outros países na administração pública, saúde, educação, agricultura, meio ambiente, energia e pequenas empresas (Kickbusch; Buss, 2011). $O$ estreitamento das relações pode gerar benefícios mútuos em negociações pacíficas e a construção de alianças para além da saúde.

Collins et al. (2012) descreveram que o plano de emergência do ex-presidente dos EUA Barack Obama para combater a epidemia de aids é um dos carros-chefe das ações diplomáticas norte-americanas. Em um cenário econômico mundial adverso, o desenvolvimento de ações diplomáticas em saúde tem se configurado como uma boa oportunidade para melhorar as relações entre países com uma boa relação custo-benefício, além de contribuir para a segurança nacional em saúde global (Boetig, 2012). Entretanto, as ações são voltadas para resolver problemas de curto prazo e não melhoram a capacidade de resposta local a médio e longo prazos (Reaves; Termini; Burkle, 2014). Os investimentos em programas de saúde globais têm função diplomática, atenuando a tensão em áreas de conflito ou pós-conflito com uma boa relação custo-benefício (Kevany et al., 2014).

Desde a institucionalização da cooperação internacional na década de 1950 até os dias atuais, países industrializados têm buscado abordar e 
resolver questões globais de pobreza e exclusão social (Almeida et al., 2010). 0 modelo tradicional de cooperação internacional é caracterizado pela ajuda humanitária de países desenvolvidos a países em desenvolvimento, unilateral, verticalizado e com predominância das visões políticas e práticas dos governos mais fortes economicamente ou das organizações não governamentais, filantrópicas e empresariais dessas nações (Buss; Ferreira, 201ob). Entretanto, modelos alternativos de cooperação internacional em saúde estão em desenvolvimento na América do Sul, através das experiências da cooperação Sul-Sul.

Essa nova abordagem de cooperação internacional em saúde tem sido denominada de "cooperação estruturante em saúde”, tendo como características a horizontalidade dos acordos e intervenções, construção de parcerias, intercâmbio de experiências, aprendizado conjunto, compartilhamento de resultados e responsabilidades. Esse novo paradigma inova em dois aspectos em comparação ao modelo tradicional de cooperação internacional em saúde: integra formação de recursos humanos, promove o fortalecimento organizacional e desenvolvimento institucional e rompe com a tradicional transferência passiva de conhecimentos e tecnologias (Almeida et al., 2010).

A cooperação Sul-Sul no Brasil é um exemplo de “cooperação estruturante para a saúde”, pois baseia-se, fundamentalmente, no fortalecimento dos sistemas locais de saúde a partir da potencialização das capacidades e recursos endógenos em cada país (Buss, 2011). No Brasil várias instituições e atores atuam no âmbito da cooperação internacional em saúde nessa perspectiva. A criação da União de Nações Sul-Americanas (Unasul) e o Plano Estratégico de Cooperação em Saúde da Comunidade de Países de Língua Portuguesa (CPLP) são experiências decorrentes do aprimoramento do diálogo diplomático estabelecido em iniciativas de integração regional na América do Sul (Mercosul, Comunidade Andina e Organização do Tratado da Cooperação Amazônica) (Buss; Ferreira, 2010a). Os principais objetivos dessas organizações são fortalecer os sistemas e serviços de saúde e suas instituições estruturantes e avançar no desenvolvimento de recursos humanos para a saúde.
As estratégias dos países para fortalecer a DSG e a cooperação internacional promovem a paz entre as nações com boa relação custo-benefício, por meio da participação das ONGs internacionais. As ações humanitárias melhoram problemas de curto prazo e auxiliam países em situação de conflito e em casos de desastre. Capacitar os recursos humanos para fortalecer os sistemas universais de saúde e desenvolver resultados de saúde sustentáveis a médio e longo prazo devem ser um dos objetivos dessas estratégias, a fim de colaborar para emancipar países emergentes.

\section{Diplomacia da saúde e o fortalecimento da saúde global}

Esforços multilaterais para desenvolver programas estratégicos que reduzam a probabilidade de futuras ameaças criam a capacidade de informações integradas entre os países (Ratzen, 2005). Nessa perspectiva, a visão de DSG dos EUA mostra que é necessário elaborar políticas globais para enfrentar os problemas de saúde, incluindo o HIV/aids e a pandemia do HiN1.

Data de 1995 a primeira estratégia internacional dos EUA sobre HIV/aids. Em 5 de maio de 2009, o então presidente Barack Obama anunciou a Iniciativa de Saúde Global para resolver alguns dos problemas mais graves de saúde que o mundo enfrenta. A experiência em lidar com o HIV/aids e o H1N1 mostra, claramente, que enfrentar os desafios da saúde global com estratégias de diplomacia e de desenvolvimento é essencial, pois a saúde dos norte-americanos está entrelaçada à saúde do resto do mundo (Jones, 2010; Daulaire, 2012). Segundo Kevany et al. (2012), o sucesso da implementação de adaptações específicas locais (religiosas, dialéticas, políticas, ambientais e de infraestrutura) tem implicações para a intervenção tanto do consumo de serviços quanto da perspectiva de DSG.

A DSG, mediante parcerias entre Estado e organizações não governamentais, é um potente instrumento para garantir o sucesso das intervenções na saúde global e gerar resultados de saúde sustentáveis e redes de vigilância em saúde global entre as nações. 0 sucesso das intervenções depende da adaptação a normas culturais, religiosas, sociais locais e de segurança da equipe. 


\section{Saúde como um bem comum entre as nações}

A partir de 1981, o posicionamento adotado em acordos internacionais foi a estratégia global Saúde Para Todos, em 200o. Entretanto, em uma primeira etapa, as diretrizes concentraram-se nas dimensões estruturais da saúde. Atualmente, os acordos internacionais baseiam-se na abordagem dos seguintes processos: saúde para todos; promoção da saúde; saúde em todas as políticas; desenvolvimento da saúde; diplomacia da saúde; e integração da saúde (Cianciara; Wysocki, 2011).

A DSG contribui para melhorar o acesso universal, a equidade e diminuir os gastos em saúde, podendo também ajudar na redistribuição de renda de acordo com o estágio de desenvolvimento de cada país e a estabilização das sociedades. Ajudar outros países a resolverem esses desafios globais de saúde contribui para o crescimento sustentável da economia global (Abe, 2013) - um exemplo são os programas de inclusão social desenvolvidos pelos EUA. Além de melhorar a qualidade de vida da população abrangida, contribui para o desenvolvimento econômico dos países (Durocher; Lord; Defranco, 2012).

Por fim, Kickbusch e Szabo (2014) reforçaram o conceito de saúde como um bem comum entre as nações, que redefine o papel do Estado em um período de globalização, comercialização e individualização.

\section{Considerações finais}

A produção científica sobre o tema é recente e abrange o campo teórico-conceitual. A DSG é uma ferramenta para promover a saúde global e nacional que, mediante parcerias entre governos e organizações não governamentais, influencia na política externa, na economia e no desenvolvimento das nações. ADSG melhora a equidade e gera cooperação internacional para resolver e priorizar problemas comuns de saúde, com o intuito de alcançar resultados de saúde sustentáveis. Em outra perspectiva, colabora para disseminar o conceito de que a saúde é um bem comum para as nações e redefine o papel do Estado nos cenários político, econômico e social mundiais. Para atingir esses objetivos, precisa promover a cooperação internacional em pesquisa, a fim de divulgar estratégias dos países para forta- lecer a saúde global, diminuir as desigualdades e estabelecer uma rede de vigilância em saúde global entre as nações.

A formação e a capacitação de recursos humanos para atuar na DSG, o desenvolvimento de resultados de saúde sustentáveis a médio e longo prazo, a adaptação cultural e a segurança das equipes que desempenham atividades em outros países, a promoção da saúde bucal global e a emancipação de países emergentes com base em intervenções feitas são grandes desafios no âmbito da DSG.

\section{Referências}

ABE, S. Japan's strategy for global health diplomacy: why it matters. The Lancet, London, v. 382, n. 9896, p. 915-916, 2013.

ADAMS, V.; NOVOTNY, T. E.; LESLIE, H. Global health diplomacy. Medical Anthropology, Pleasantville, v. 27, n. 4, p. 315-323, 2008.

ALMEIDA, C. A. A experiência da Fiocruz na formação de profissionais em saúde global e diplomacia da saúde: base conceitual, estrutura curricular e primeiros resultados. Reciis, Rio de Janeiro, v. 4, n. 1, p. 148-164, 2010.

ALMEIDA, C. et al. A concepção brasileira de “cooperação Sul-Sul estruturante em saúde”. Reciis, Rio de Janeiro, v. 4, n. 1, p. 25-35, 2010.

BADEAU, J. S. Diplomacy and medicine. Bulletin of the New York Academy of Medicine, New York, v. 46, n. 5, p. 303-312, 1970.

BARDIN, L. Análise de conteúdo. São Paulo: Edições 70, 2011.

BARRACLOUGH, S.; PHUA, K. L. Health imperatives in foreign policy: the case of Malaysia. Bulletin of the World Health Organization, Geneva, v. 85, n. 3, p. 225-229, 2007.

BERNARDINI-ZAMBRINI, D. A.; PEDROSA, F.; SOTELO, J. M. Global health, diplomacy, and the case of the "killer cucumbers". Revista Panamericana de Salud Publica, Washington, DC, v. 3o, n. 5, p. 501-502, 2011.

BOETIG, B. J. Bilateral institutional relationships: a new mission for U.S. DoD medical capabilities 
in support of health diplomacy. Military Medical, London, v. 177, n. 7, p. 763-765, 2012.

BUSS, P. M. Brazil: structuring cooperation for health. The Lancet, London, v. 377, n. 9779, p. 17221723, 2011.

BUSS, P. M. Global health and health diplomacy. Cadernos de Saúde Pública, Rio de Janeiro, v. 29, n. 1, p. 1-2, 2013.

BUSS, P. M.; FERREIRA, J. R. Diplomacia da saúde e cooperação Sul-Sul: as experiências da Unasul saúde e do Plano Estratégico de Cooperação em Saúde da Comunidade de Países de Língua Portuguesa (CPLP). Reciis, Rio de Janeiro, v. 4, n. 1, p. 106-118, 2010a.

BUSS, P. M.; FERREIRA, J. R. Ensaio crítico sobre a cooperação internacional em saúde. Reciis, Rio de Janeiro, v. 4, n. 1, p. 93-105, 2010b.

CAHILL, K. M. Health and foreign policy: an American view. Annals of Tropical Medicine and Parasitology, London, v. 91, n. 7, p. 735-741, 1997.

CHALKIDOU, K.; VEGA, J. Sharing the British National Health Service around the world: a selfinterested perspective. Global Health, London, v. 9, n. 2, p. 1-10, 2013.

CIANCIARA, D.; WYSOCKI, M. J. Position of health at international relations. Part II. Organizational dimensions of health. Przegla, epidemiologiczny, Warszawa, v. 65, n. 2, p. 379-387, 2011.

COLLINS, C. et al. Four principles for expanding PEPFAR's role as a vital force in US health diplomacy abroad. Health Affairs, Millwood, v. 31, n. 7, p. 1578-1584, 2012.

DAULAIRE, N. The Global Health Strategy of the Department of Health and Human Services: building on the lessons of PEPFAR. Health Affairs, Millwood, v. 31, n. 7, p. 1573-1577, 2012.

DRAGER, N.; FIDLER, D. P. Foreign policy, trade and health: at the cutting edge of global health diplomacy. Bulletin of the World Health Organization, Geneva, v. 85, n. 3, p. 162, 2007.

DUROCHER, J.; LORD, J.; DEFRANCO, A. Disability and global development. Disability and Health Journal, New York, v. 5, n. 3, p. 132-135, 2012.
FEDOROFF, N. V. Science diplomacy in the 21st century. Cell, Cambridge, v. 136, n. 1, p. 9-11, 2009.

FEINSILVER, J. M. Fifty years of Cuba's medical diplomacy: from idealism to pragmatism. Cuban Studies, Pittsburgh, v. 41, p. 85-104, 2010.

FIDLER, D. P. The globalization of public health: the first 100 years of international health diplomacy. Bulletin of the World Health Organization, Geneva, v. 79, n. 9, p. 842-849, 2001. FOURIE, P. Turning dread into capital: South Africa's AIDS diplomacy. Globalization and Health, London, v. 9, n. 8, p. 1-12, 2013.

FRENCH, H. F. From discord to accord. World Watch, Washington, DC, v. 5, n. 3, p. 26-32, 1992.

GARCIA, I.; TABAK, L. A. Global oral health inequalities: the view from a research funder. Advances in Dental Research, Washington, DC, v. 23, n. 2, p. 207-210, 2011.

HARDIMAN, M. The revised International Health Regulations: a framework for global health security. International Journal of Antimicrobial Agents, Amsterdam, v. 21, n. 2, p. 207-211, 2003.

JONES, K. A. New complexities and approaches to global health diplomacy: view from the U.S. Department of State. PLoS Medicine, San Francisco, v. 7, n. 5, p. e10oo276, 2010.

KEVANY, S. et al. Health diplomacy the adaptation of global health interventions to local needs in Sub-Saharan Africa and Thailand: evaluating findings from Project Accept (HPTN o43). BMC Public Health, London, v. 12, n. 459, 2012.

KEVANY, S. et al. Global health diplomacy in Iraq: international relations outcomes of multilateral tuberculosis programmes. Medicine, Conflict and Survival, London, v. 30, n. 2, p. 91-109, 2014.

KICKBUSCH, I. Global health diplomacy: how foreign policy can influence health. British Medical Journal, London, v. 342, p. d3154, 2011. KICKBUSCH, I.; BUSS, P. Global health diplomacy and peace. Infectious Disease Clinics of North America, Philadelphia, v. 25, n. 3, p. 601-610, 2011. 
KICKBUSCH, I. et al. Global health diplomacy: training across disciplines. World Hospital Health

Services, London, v. 43, n. 4, p. 20-22, 2007.

KICKBUSCH, I.; KÖKÉNY, M. Global health diplomacy: five years on. Bulletin of the World Health Organization, Geneva, v. 91, n. 3, p. 159, 2013.

KICKBUSCH, I.; SZABO, M. M. C. A new governance space for health. Global Health Action, London, v. 7, p. 17, 2014.

KOSARAJU, A. et al. Use of mobile phones as a tool for United States health diplomacy abroad. Telemedicine and e-Health, Larchmont, v. 16, n. 2, p. 218-222, 2010.

NOVOTNY, T. E. US Department of Health and Human Services: a need for global health leadership in preparedness and health diplomacy. American Journal of Public Health, Washington, DC, v. 96, n. 1, p. 1113, 2006.

ÖZDEMIR, V. et al. Ready to put metadata on the post-2015 development agenda? Linking data publications to responsible innovation and science diplomacy. Omics, Los Angeles, v. 18, n. 1, p. 1-9, 2014.

RATZAN, S. C. Innovation in the post-MDG environment: advancing global health diplomacy in pursuit of the global good. Journal of Health
Communication, London, v. 18, n. 5, p. 479-484, 2013.

RATZEN, S. C. Beyond the 2004 tsunami: health diplomacy as a response. Journal of Health Communication, London, v. 10, p. 197-198, 2005.

REAVES, E. J.; TERMINI, M.; BURKLE, F. M. Reshaping US Navy Pacific response in mitigating disaster risk in South Pacific Island nations: adopting community-based disaster cycle management. Prehospital Disaster Medicine, Solana Beach, v. 29, n. 1, p. 6o-68, 2014.

RØTTINGEN, J. A. et al. Global-health research architecture - time for mergers? The Lancet, London, v. 373, n. 9659, p. 193-195, 2009.

SEIFF, A. Indonesia's year for global health diplomacy. The Lancet, London, v. 382, n. 9889, p. 297, 2013.

SHARPLESS, J. World population growth, family planning, and American foreign policy. Journal of Policy History, Cambridge, v. 7, n. 1, p. 72-102, 1995 .

VANTI, N. A. P. Da bibliometria à webometria: uma exploração conceitual dos mecanismos utilizados para medir o registro da informação e a difusão do conhecimento. Ciência da Informação, Brasília, DF, v. 31, n. 2, p. 152-162, 2002.

\section{Contribuição dos autores}

Martins foi responsável pela concepção, análise e interpretação dos dados. Aguiar realizou a concepção, revisão crítica e aprovação da versão a ser publicada. Mesquita, Alexandrino, Silva e Moreno realizaram a coleta e análise dos dados. Todos os autores contribuíram com a redação do manuscrito.

Recebido: 05/09/2016

Reapresentado: 07/02/2017

Aprovado: 10/02/2017 